\title{
Spin test of three-dimensional composite rotor using polymer ring as a connection device for high-speed flywheel
}

\author{
Noboru HIROSHIMA*, Hiroshi HATTA ${ }^{*, * *}$, Yuuichi NAGURA ${ }^{* * *}$, Masashi KOYAMA ${ }^{* * * *}$, \\ Takenobu SAKAI ${ }^{* * * * *}$ and Yasuo KOGO ${ }^{* * *}$ \\ *Department of Space and Astronautical Science, School of Physical Sciences, \\ SOKENDAI (The Graduate University for Advanced Studies), Sagamihara, 252-0022, Japan \\ E-mail : hiroshima.noboru@keh.biglobe.ne.jp \\ **Institute of Space and Astronautical Science, Japan Aerospace Exploration Agency, Sagamihara, 252-0022, Japan \\ *** Department of Materials Science and Technology, Tokyo University of Science, Noda, 278-8510, Japan \\ ${ }^{* * * \star}$ Department of Interdisciplinary Science and Engineering, Meisei University, Hino, 191-8506, Japan

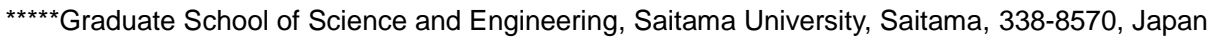

\section{Received 25 April 2016}

\begin{abstract}
A method for connecting a driving shaft to a three-dimensional composite disk via a polymer ring was examined to achieve stable rotation at high rotation speeds. Polyoxymethylene (POM) was adopted as the polymer ring material. Compression test was conducted to determine the limit of linear viscoelasticity, and creep resistance of POM was examined to evaluate its long-term durability. Structural design using finite element analysis including long-term creep resistance has shown that this method was predicted to be effective up to a tip speed of 1210 $\mathrm{m} / \mathrm{s}$. A three-dimensional composite disk was reinforced to three directions in accordance with the cylindrical coordinates. A trial composite disk with outer and inner diameters of 304 and $41 \mathrm{~mm}$, respectively, was manufactured and tested up to a tip speed of $908 \mathrm{~m} / \mathrm{s}$. Vibration amplitudes were measured using gap and laser sensors. The vibration phenomenon and methods of suppressing vibration were discussed for higher rotation speeds.
\end{abstract}

Key words : Carbon fiber reinforced plastics, 3-Dimensional reinforcement, Compression test, Viscoelasticity, Creep, Finite element analysis (FEA), Resin transfer molding (RTM), Vibration

\section{Introduction}

Carbon fiber reinforced plastics (CFRPs) have been often applied to flywheel rotors for electric energy storage systems in order to achieve high-speed rotation by exploiting its high specific strength (Ha, 2006, Arvin, 2006). Cooling or press fitting is generally used to fix a disk with a shaft via some device (hub) (Ha, 2006, Arvin, 2006, Hayes, 1998, Takahashi, 2002, Nasu, 2002). This connection produces compressive stresses on the disk-hub interface, and these stresses in turn make the connection between the two parts stable. However, as rotational speed increases, the disk is subjected to greater centrifugal force than the hub (or shaft). Consequently, the disk tends to detach from the shaft, inducing rotational vibration. Vibration growth at high-speed rotation affected or suspended spin test (Ha, 2006, Takahashi, 2002). The connection between the disk and hub was considered to be important (Arvin, 2006, Yasser, 2003).

We have developed an optimum design of a three-dimensional carbon fiber composite (3DCF) disk in which reinforcement is distributed along the axial directions of the cylindrical coordinate system $(r, \theta, z)$ and shown that high energy density including the hub can be stored, especially when the ratio of the inner to the outer radius of the disk, $\lambda=$ $r_{1} / r_{2}$, is less than 0.5 (Hiroshima, 2015). However, to effectively use the optimized composite disk, a countermeasure against the detachment of the disk and hub (or shaft) and therefore against the resulting vibration should be developed (Hiroshima, 2016). The development of such a stable rotor assembly under high speed rotation including the disk, hub and shaft is the objective of the present study.

To obtain a rotor assembly with $\lambda$ smaller than 0.5 , Gowayed et al. (2003) devised a box hub mounted between the 
disk with $\lambda$ of 0.436 and shaft and numerically showed that $93 \mathrm{Wh} / \mathrm{kg}$ of the maximum energy density including the hub can be attained. Gabrys et al. (1997) reported a composite disk with a $\lambda$ of 0.075 by placing three interlayers of elastomers between multiple concentric composite rings. At maximum speed, positive radial stress in the interlayers mean that elastomers are elongated not to separate from neighboring composite rings. The maximum energy density of this disk including the shaft was measured experimentally to be $84 \mathrm{Wh} / \mathrm{kg}$. However, in their study, time-dependent material properties were not discussed for long-term durability.

We have developed a trial 3DCF disk with a $\lambda$ of 0.13 and evaluated three methods for connecting the disk to the shaft (hub). Through this experimental study, we found that a 3DCF disk is likely to have a certain amount of initial mass unbalance that can cause in-plane slippage (Hiroshima, 2016). We concluded that the method of placing a polyoxymethylene (POM) ring between the disk and shaft effectively maintains their connection at high rotation speeds. When cooling fitted, polymers can thus produce a large deformation mismatch by high thermal expansion coefficients and thus maintain compressive stresses on the disk-hub interface up to high rotation speeds. However, polymers exhibit notable creep and stress relaxation behavior. Hence, achieving stable high-speed rotation necessitates considering timedependent properties when a polymer is used as a connection device.

In this study, we demonstrate the effectiveness of connecting a 3DCF disk to a shaft using a POM ring as a hub. First, POM was subjected to compression tests to determine the allowable stress for cooling fit (deformation mismatch) within the limits of elastic behavior. Second, on the basis of the viscoelastic test results for POM, possible deformation mismatch was predicted by finite element analysis (FEA) to maintain a stable connection. Finally, a spin test for a trial rotor was conducted. The vibration was monitored at several positions on the disk and a test fixture using laser and gap sensors to elucidate the vibration behavior and to explore vibration suppression.

\section{Nomenclature}

$\begin{array}{lll}r, \theta, z & : \text { Cylindrical coordinates } \\ V_{\mathrm{f}} & : & \text { Total volume fraction of fiber } \\ V_{\mathrm{fr}} & : & \text { Volume fraction of fiber in the radial direction } \\ V_{\mathrm{f} \theta} & : & \text { Volume fraction of fiber in the circumferential direction } \\ V_{\mathrm{fz}} & : & \text { Volume fraction of fiber in the axial direction } \\ r_{1}, r_{2} & : & \text { Inner and outer radii, respectively [mm] } \\ r_{\mathrm{h}} & : & \text { Outer radius of a hub [mm] } \\ \lambda & : & r_{1} / r_{2} \\ E & : & \text { Elastic modulus [Pa] } \\ E_{\mathrm{L}} & : & \text { Longitudinal elastic modulus [Pa] } \\ E_{\mathrm{T}} & : & \text { Transverse elastic modulus [Pa] } \\ v_{\mathrm{LT}} & : & \text { Poisson's ratio relating contraction in the } T \text { direction as a result of extension in the } L \text { direction } \\ v_{\mathrm{TT}} & : & \text { Poisson's ratio relating contraction in the } T \text { direction as a result of extension in the } T \text { direction } \\ G & : \text { Shear modulus [Pa] } \\ A & : \text { Thermal expansion of coefficient [ } \mu / \mathrm{K}] \\ \sigma_{\mathrm{r}}, \sigma_{\theta} & : \text { Normal stress in radial and circumferential directions, respectively [Pa] } \\ \omega & : \text { Angular velocity [rad/sec] } \\ \delta & : \text { Amplitude induced by asymmetric deformation of } 3 \mathrm{DCF} \text { disk [mm] } \\ \delta_{\mathrm{FIT}} & : \text { Deformation mismatch induced by cooling or press fit [mm] } \\ \delta_{\mathrm{FIT}, \mathrm{c}} & : \text { Deformation mismatch induced by cooling fit [mm] }\end{array}$

\section{Design}

\subsection{3-dimensional fabric design and composite manufacturing}

A rotation-tested 3DCF disk was reinforced to three directions in accordance with the cylindrical coordinates ( $r, \theta$, z) (Hiroshima, 2015). Figure 1(a) shows an optimized disk fabricated in our previous study (Hiroshima, 2015). The thickness of the 3DCF disk decreases in inverse proportion to the radius. In this disk, the fiber volume fraction in the $r$ direction $\left(V_{\mathrm{fr}}\right)$ is kept constant because continuous fiber bundles run straight from the inner to the outer radius. This disk 
has an in-plane volume fraction of fiber of $0.45\left(V_{\mathrm{fr}}+V_{\mathrm{f} \theta}\right)$ and an axial volume fraction of fiber of $0.02\left(V_{\mathrm{fz}}\right)$ and was predicted to have a burst tip speed of $1592 \mathrm{~m} / \mathrm{s}$.

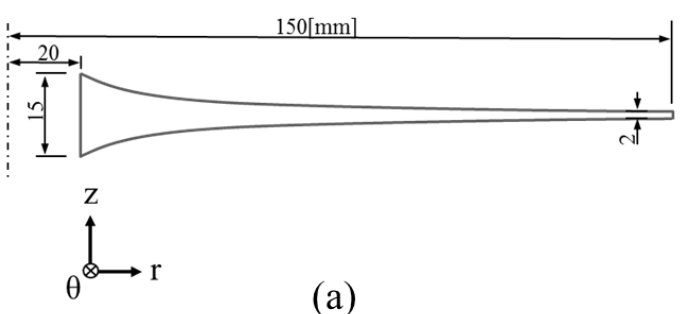

(a)

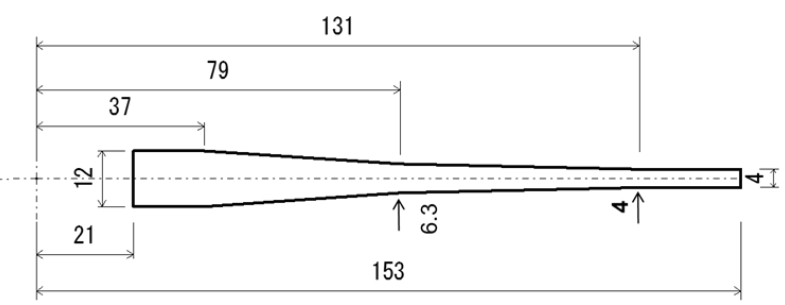

(b)

Fig. 1 A right half cross-section of the 3DCF disk: (a) optimized disk $\left(\mathrm{V}_{\mathrm{fr}} / \mathrm{V}_{\mathrm{ff}} / \mathrm{V}_{\mathrm{fz}}=0.186 / 0.264 / 0.02\right)$, and (b) modified trail disk $\mathrm{V}_{\mathrm{fr}} / \mathrm{V}_{\mathrm{ff}} / \mathrm{V}_{\mathrm{fz}}=0.183 / 0.252 / 0.024$ on average).

Figure 1(b) shows an actually fabricated disk that was modified for manufacturing convenience. This disk has $V_{\text {fr }}$ and $V_{\mathrm{f} \theta}$ values of 0.183 and 0.252 on average, respectively (Hiroshima, 2016). The configuration of the optimized disk shown in Fig. 1(a) may cause several problems in fabrication. To circumvent these problems, the disk configuration was modified as follows.

1) To avoid distortion and deformation after curing, the thickness at the outer radius was increased from 2 to 4 $\mathrm{mm}$. This change has another advantage of improving the energy density of the disk.

2) The thickness profile was simplified and varied to four straight lines.

3) Flat and horizontal planes were formed from the inner radius to a circle of $r=37 \mathrm{~mm}$ for precise positioning of the disk in the assembly system.

The stress distribution in the disk shown in Fig. 1(b) was calculated using the ABAQUS commercial software (ver. 6.2). The predicted burst tip speed and energy density including the hub were $1520 \mathrm{~m} / \mathrm{s}$ and $121 \mathrm{Wh} / \mathrm{kg}$, respectively (Hiroshima, 2016).

The reinforcing fabric was composed of high strength type PAN-based-carbon-fiber (Torayca T1000G; Toray Co. Ltd., Japan) for $r$ and $\theta$ bundles, and low-strength type (Torayca T300; Toray Co. Ltd., Japan) for $z$ bundles because of the small stress induced in the axial direction. Shikibo Ltd., Japan supplied this fabric. Table 1 presents the material properties of the fiber, resin, and steel (for assembling fixture) used in stress analyses. The composite disk was formed by resin transfer molding. Cyanate ester-type resin of $180^{\circ} \mathrm{C}$ cure type (EX-1545; Koninklijke Ten Cate nv.) was used for the matrix. In the composite fabrication process, the fabric was placed in the empty cavity of the metal-matched die having the same dimensions as shown in Fig. 1(b). The resin was infused into the die cavity under reduced pressure (Hiroshima, 2016, Yoshimura, 2010).

Table 1 Physical and elastic properties of carbon fiber, matrix resin, POM and steel

\begin{tabular}{llllllrr}
\hline Material & $\begin{array}{l}\text { Mass } \\
\text { density } \\
\rho\left(\mathrm{kg} / \mathrm{m}^{3}\right)\end{array}$ & $\begin{array}{l}\text { Longitudinal elastic } \\
\text { modulus } \\
E_{L}(\mathrm{GPa})\end{array}$ & $\begin{array}{l}\text { Transverse elastic } \\
\text { modulus } \\
E_{T}(\mathrm{GPa})\end{array}$ & $\begin{array}{l}\text { Thermal expansion } \\
\text { coefficient } \\
\alpha(\mu / \mathrm{K})\end{array}$ & $\begin{array}{l}\text { Poisson's } \\
\text { ratio }\end{array}$ & $\begin{array}{l}\text { Tensile } \\
v_{\mathrm{LT}}\end{array}$ & $\begin{array}{l}v_{T T} \\
\text { strength } \\
(\mathrm{MPa})\end{array}$ \\
\hline T1000G & $1800^{\mathrm{a}}$ & $294^{\mathrm{a}}$ & $40^{\mathrm{b}}$ & - & $0.31^{\mathrm{b}}$ & $0.33^{\mathrm{b}}$ & $6370^{\mathrm{a}}$ \\
T300 & $1760^{\mathrm{a}}$ & $230^{\mathrm{a}}$ & $40^{\mathrm{b}}$ & - & $0.31^{\mathrm{b}}$ & $0.33^{\mathrm{b}}$ & $3530^{\mathrm{a}}$ \\
matrix resin & 1250 & 3.4 & - & - & 0.36 & - & $\mathrm{NA}$ \\
POM & 1400 & 2.5 & - & 110 & 0.35 & - & 63 \\
SNCM616 & 7800 & 206 & - & - & 0.3 & - & $1180^{\mathrm{c}}$ \\
\hline
\end{tabular}

a Toray carbon fiber America, T1000G, T300 data sheet

b (Hiroshima, 2015)

c JIS G4103, ultimate strength

\subsection{Assembly and design concept of the joint}

Figure 2 shows test fixtures used for the specimen. To maintain accurate coaxiality during the assembly, the structure in which a shaft penetrates the center of the POM ring was adopted. The 3DCF disk is fixed to the shaft by a nut. A POM ring was positioned between the 3DCF disk and shaft, and compressive strain was induced on the interface via a cooling and press fit process using liquid nitrogen. The outer radii of the shaft, POM ring, and 3DCF disk were 11, 21, and 153 $\mathrm{mm}$, respectively. The compressive stress induced by the cooling and press fit process at the POM ring-disk interface 
was determined using FEA. SNCM616 was used as the shaft material (Hiroshima, 2016).

POM (CP-15X, Polyplastic Co., Ltd.,) with high creep resistance was used as the resin ring. Table 1 shows POM properties. Generally, plastic materials have high thermal expansion coefficients compared with those of metals. Thus, a large deformation (compressive strain) can be induced by the cooling fit process. The two parts remain connected as long as the cooling-fit-induced compressive strain is not canceled by the tensile deformation of the disk under the centrifuge force of high-speed rotation (Hiroshima, 2016).

The displacement gained by the cooling fit $\delta_{\mathrm{FIT}, \mathrm{c}}$ is expressed as

$$
\delta_{\mathrm{FIT}, \mathrm{c}}=r_{\mathrm{h}} \Delta T \alpha,
$$

where $r_{\mathrm{h}}$ is the outer radius of the hub to be cooling-fitted, $\Delta T$ is the temperature difference between room temperature and the cooling fit temperature, and $\alpha$ is the thermal expansion coefficient of the hub. A potential fitting displacement of $0.462 \mathrm{~mm}$ is obtained by assuming a POM $\alpha$-value of $110 \mu / \mathrm{K}$ (Table 1) in eq. (1) under the assumption of liquid nitrogen as the cooling medium $(\Delta T=200)$ and the outer radius of POM ring $\left(r_{\mathrm{h}}=21 \mathrm{~mm}\right)$. The displacement of the inner radius of the 3DCF disk at the burst tip speed of $1520 \mathrm{~m} / \mathrm{s}$ is calculated to be $0.41 \mathrm{~mm}$ by FEA, which is smaller than the fitting displacement of $0.462 \mathrm{~mm}$ calculated using eq. (1). Thus, when POM is used as the ring material, the connection (joint) is expected to be maintained at a high burst tip speed of $1520 \mathrm{~m} / \mathrm{s}$.

In the assembly process, the POM ring was first cooling fitted into the 3DCF disk. After the POM ring was cooled and shrunk by liquid nitrogen, it was inserted into the center hole of the 3DCF disk. Then, in the temperature recovery process of the POM ring to room temperature, compressive stress was induced at the ring-disk interface. The shaft was then press fitted into the center hole of the POM ring at a rate of $0.05 \mathrm{~mm} / \mathrm{min}$ using a screw-driven mechanical testing machine (Autograph AG-5000A; Shimadzu Corp.).

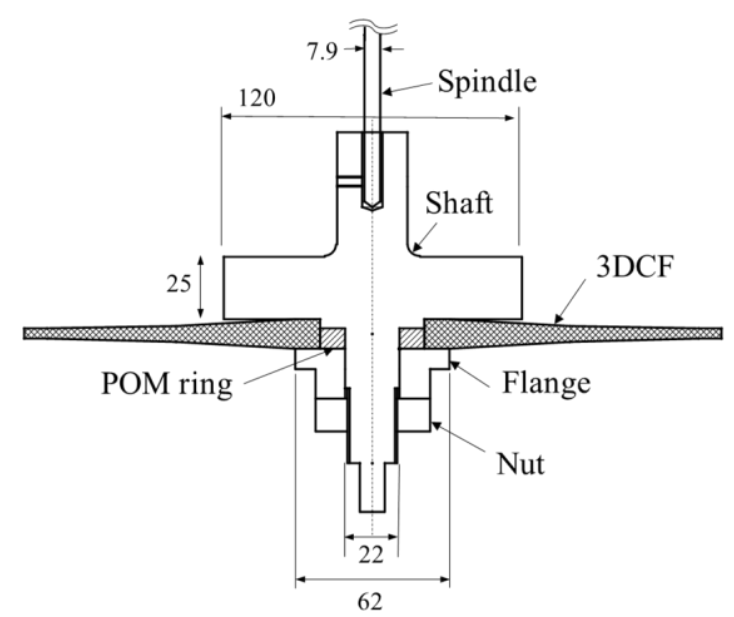

(a)

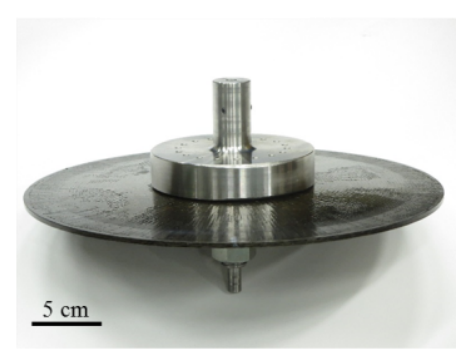

(b)

Fig. 2 Test fixtures for spin tests in which POM ring is inserted between 3DCF and shaft: (a) assembled fixtures, (b) outlook of the specimen with the fixtures.

\section{Experiments}

\subsection{Spin tests}

The rotational mass unbalance in the disk was investigated using a dynamic balancer (FH-216; Mitutoyo Corp.). The 3DCF disk shown in Fig. 1(b) has a mass unbalance of $19.3 \mathrm{~g} \cdot \mathrm{mm}$ after the curing process. After spin test rotor was assembled as shown in Fig. 2(a), the entire mass unbalance was adjusted to $0.15 \mathrm{~g} \cdot \mathrm{mm}$ by adding small screws at the small holes engraved into the shaft. This residual unbalance was on a level of grade G1 according to the balance quality grade (JIS 0905, 1992), thereby allowing a maximum rotation speed of 100,000 rpm.

Residual unbalance can also be improved and adjusted by grinding the 3DCF disk. However, this can damage the fiber bundles just underneath the disk surface and cause them to fly away, which could result in further mass unbalance (Goto, 2003). Therefore, we did not adopt this method. 
Spin tests were conducted using a spin tester (Maruwa Co. Ltd., Japan) driven by an air turbine (Fig. 3). During the tests, the chamber pressure was reduced to $80 \mathrm{~Pa}$. The rotation speed was increased intermittently at $100 \mathrm{rpm} / \mathrm{s}$. As shown in Fig. 4, displacement sensors were placed at four points: U, L, P, and Q. point $\mathrm{O}$ is the intersection of the disk midplane and the axial center of the shaft. Although vibration at point $\mathrm{O}$ cannot be measured directly using a sensor, its amplitude is important for understanding vibration behavior. This behavior was estimated from the radial amplitudes at $\mathrm{U}$ and L measured using eddy current sensors (PU-05; Applied Electronics Co., Japan).

The gap sensors generated large noise measuring the vibration amplitude on the 3DCF disk surface. Therefore, laser sensors (lkg-15; Keyence Co., Japan) were used to measure the amplitude of the outer radius at points P and Q. Three sensors (U, L, and P) were placed at the same angles in the circumferential direction. Except for point U, the amplitude measurements were limited to $38,000 \mathrm{rpm}$ in order to avoid sensor damage from unexpected disk fracture.

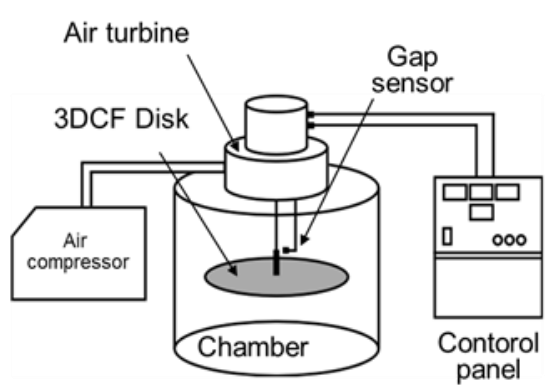

Fig. 3 Schematic diagram of the air turbine drive spin tester.

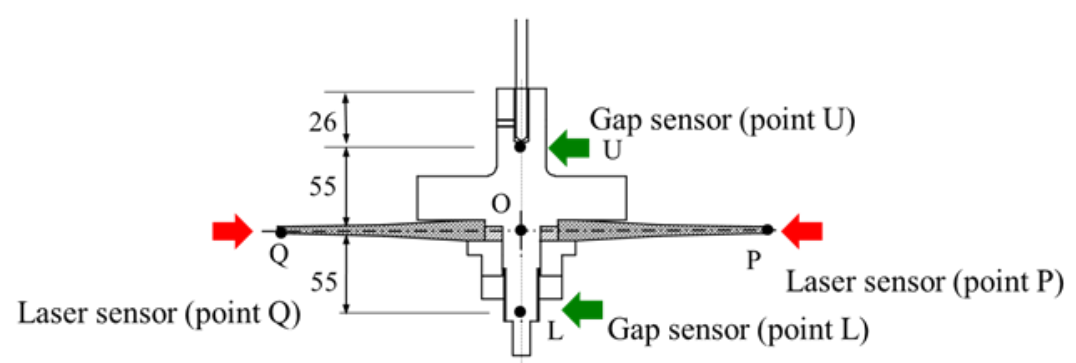

Fig. 4 Positions of displacement measurements. Three sensors measuring at $\mathrm{U}, \mathrm{L}$, and $\mathrm{P}$ were attached at the same phase angle in the circumferential direction.

\subsection{Compression tests}

A large $\delta_{\text {FIT }}$ enables high rotational speeds. However, this also produces large compressive stress in the POM ring. The compression strength of POM was measured to estimate the maximum $\delta_{\text {FIT }}$.

Compression tests were conducted for the POM ring according to standard JIS K7181 (2011). The specimen shown in Fig. 5(a) was used, and its surface area of $4 \times 10 \mathrm{~mm}^{2}$ was uniformly loaded. In accordance with the JIS standard, the dimensional accuracy of each specimen was better than $0.01 \mathrm{~mm}$, and the squareness tolerance was less than $0.025 \mathrm{~mm} / 10$ $\mathrm{mm}$. Each specimen was compressed at a rate of $0.5 \mathrm{~mm} / \mathrm{min}$ using a screw-driven mechanical testing machine. Strain gages (KFP-2-120-C1-65; Kyowa Electronic Instruments Co., Ltd.) were patched on both $10 \times 10 \mathrm{~mm}^{2}$ square surfaces. Test results were deemed acceptable when the difference in the strains was less than $5 \%$.

\subsection{Dynamic viscoelastic tests}

To evaluate the creep behavior of the POM ring for long-term use, we conducted dynamic viscoelastic tests (JIS K7244, 1998). The dynamic viscoelastic behavior was measured using a shear device (Rheometrics; RSA2). Figure 5(b) shows the dimensions of the specimens. Tests were conducted in a three-point bending mode, where a sinusoidal displacement with maximum $0.2 \%$ strain from 0.6 to $12 \mathrm{~Hz}$ was applied. Measurement temperatures were varied at $5^{\circ} \mathrm{C}$ intervals between 25 and $135^{\circ} \mathrm{C}$.

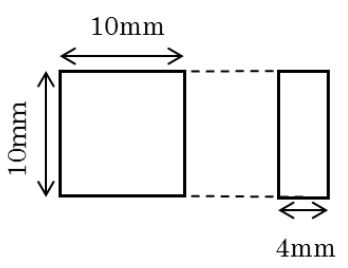

(a)

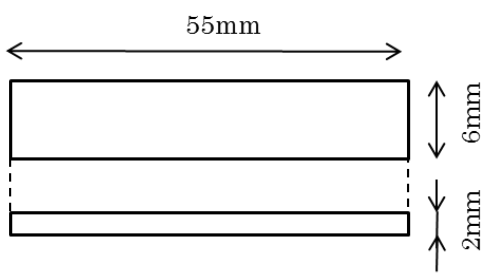

(b)

Fig. 5 Dimensions of test specimens: (a) compressive strength, (b) dynamic viscoelastic properties. 


\subsection{Contact, viscoelastic, and elastic FEAs of rotors}

FEAs were conducted using commercial software (ABAQUS, ver. 6.11). Figure 6(a) displays an analytical model including contacts between the disk, POM ring, and shaft. Eight axisymmetric rectangle node elements were used, and contact elements including friction effect were defined on all contact boundaries of the three parts. The orthogonal material properties vary in the radial direction and were estimated using the equivalent inclusion methods (Hiroshima, 2015). Table 2 presents elastic properties of composites used for computations when $V_{f r}=0.183, V_{f \theta}=0.252, V_{f z}=0.024$.

The model has 6120 elements and 18,919 nodes in total. The axial displacement was constrained on the upper surface of the shaft, as shown in Fig. 6(a). The flange shown in Fig. 2 has outer and inner diameters of 62 and $22 \mathrm{~mm}$, respectively, on its upper surface, which contacts the 3DCF disk and POM ring. A total axial force of $200 \mathrm{~N}$ was applied to the contact surface line, as shown in Fig. 6(a). Constraint of the same displacement was adopted on the contact surface line. Friction factors on the contact surfaces were set to 0.15 (Ogasawara, 2012).

The calculations consisted of the following three steps:

1) Contact analysis to determine the contact pressure $\left(\sigma_{\mathrm{r}}\right)$ after cooling fit on the POM ring-disk interface.

2) Viscoelastic analysis to predict the time-dependent contact pressure within three years at temperatures ranging from -60 to $80{ }^{\circ} \mathrm{C}$.

3) Static analysis for estimation of the maximum rotation speed at which joining can be maintained.

The temperature range in step 2 covers cold to tropical regions. The radial compressive stress decreases with time because of the time-dependent behavior of POM. This tendency reduces the maximum connection-keeping speed. The tip speed beyond which the radial stress $\left(\sigma_{\mathrm{r}}\right)$ turns positive and separation occurs was analytically calculated and defined as the maximum connection-keeping tip speed (MCKTS) possible.

There is another FEA procedure in which step 3 is performed prior to step 2. In this case, the radial compressive stress decreases due to centrifugal force. This means the MCKTS is enhanced because the viscoelastic effect also becomes weak. Thus, this FEA procedure to determine the MCKTS was neglected in this study.

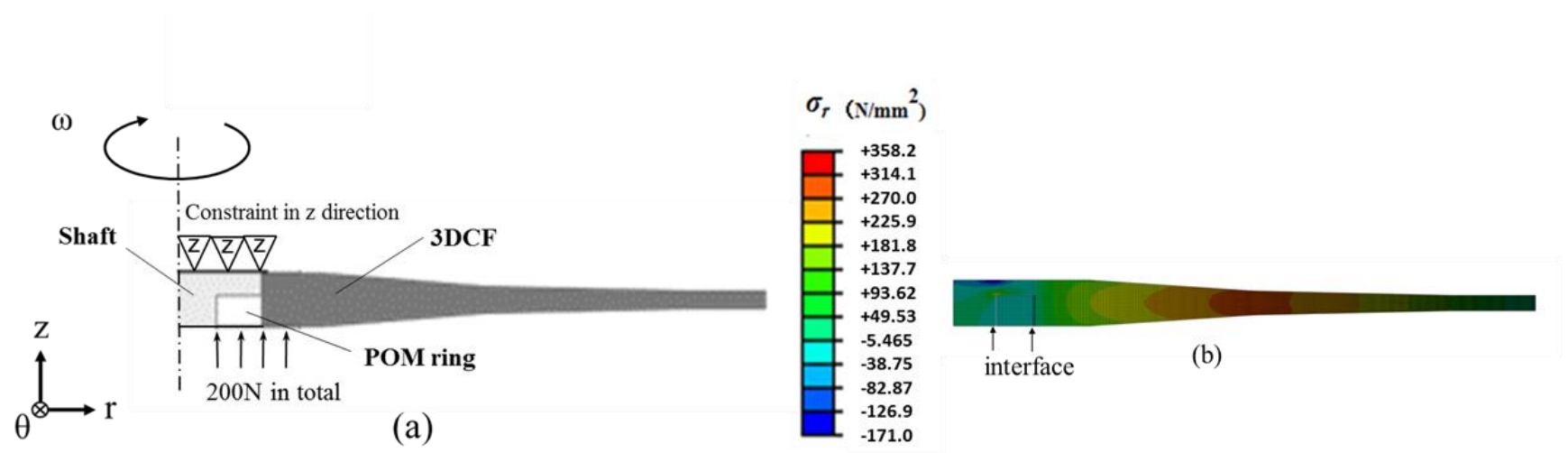

Fig. 6 A right half cross-section FEA model of the trial rotor: (a) model and

(b) calculated distribution of normal stress $\sigma_{r}$ at a tip speed of $900 \mathrm{~m} / \mathrm{s}$.

\section{Results}

\subsection{Compression tests}

Figure 7 shows the compressive stress-strain relation of POM under cyclic loading, i.e., cycling of loading/unloading/reloading under increasing maximum compressive stress. A slight permanent strain appeared at a maximum stress of $80 \mathrm{MPa}$. On the basis of this result, $\delta_{\mathrm{FIT}}$ was designed to maintain a maximum stress of less than 70 MPa. 


\subsection{Viscoelastic tests}

Figure 8 shows the time-dependent storage modulus of $\mathrm{POM}$ tested in $5^{\circ} \mathrm{C}$ intervals between 25 and $135^{\circ} \mathrm{C}$. Figure 9 shows the master curve for storage modulus of POM at a reference temperature of $25^{\circ} \mathrm{C}$ (Sakai, 2008). From this master curve, creep compliance distribution was determined on the basis of the generalized Voigt model and the result was used for analyzing the viscoelasticity via FEA.

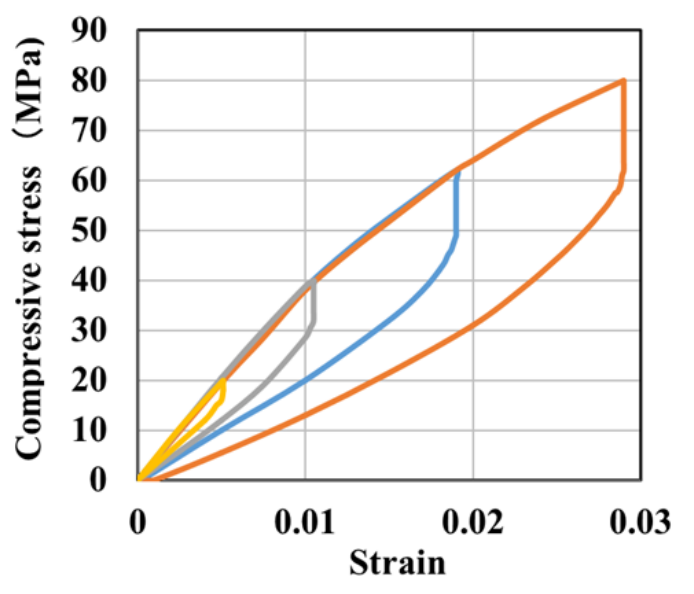

Fig.7 Compressive stress - strain relation of POM under cyclic loading.

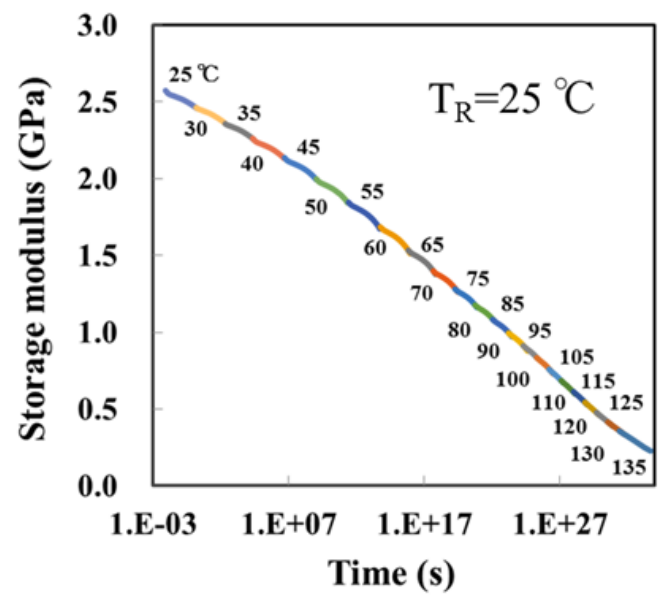

Fig.9 Mater curve for storage modulus of POM tested at 5 degree intervals between $25{ }^{\circ} \mathrm{C}$ and $135{ }^{\circ} \mathrm{C}$ (Reference Temperature $; \mathrm{T}_{\mathrm{R}}=25^{\circ} \mathrm{C}$ )

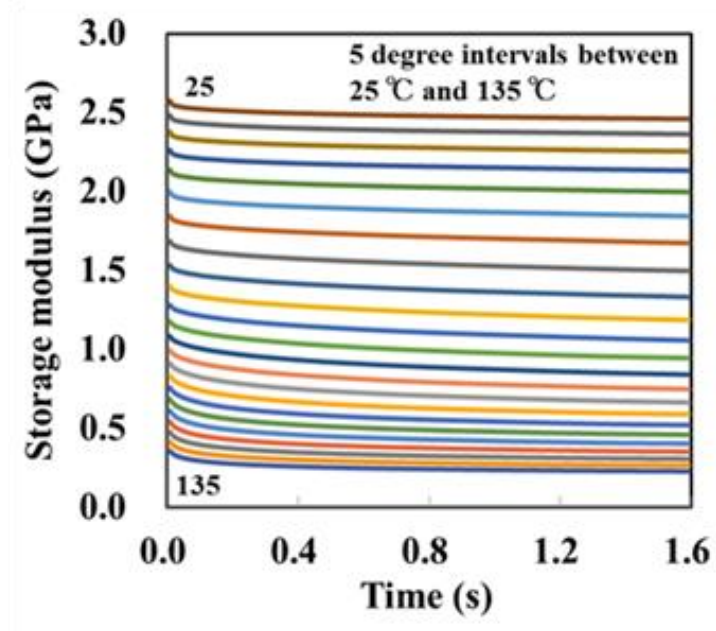

Fig.8 Time-dependent storage modulus of POM tested at 5 degree intervals between $25^{\circ} \mathrm{C}$ and $135^{\circ} \mathrm{C}$.

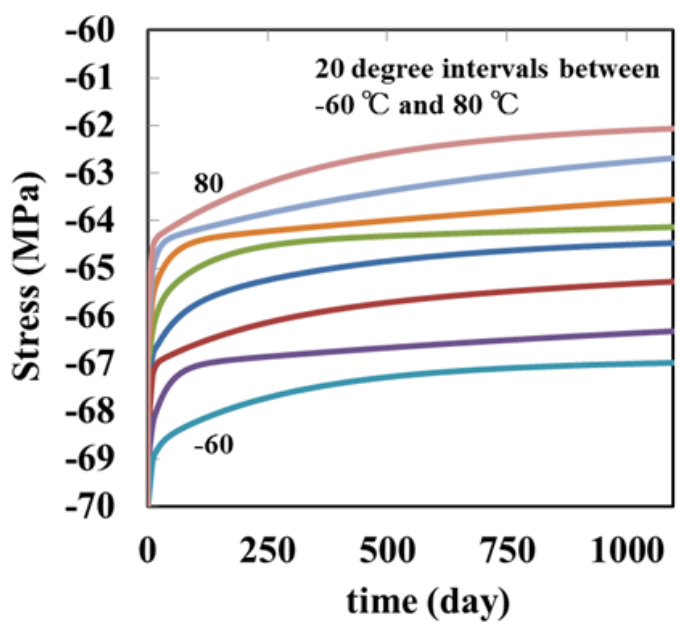

Fig.10 Time-dependent compressive stress on the POM ring-3DCF disk interface without rotation.

\subsection{Viscoelastic analysis}

First, FEA was conducted for the cooling fit process. The deformation mismatches $\left(\delta_{\mathrm{FIT}} \mathrm{s}\right)$ in the radial direction are defined as the overlapped length of the inner radius of the disk and outer radius of the POM ring or the inner radius of the POM ring and shaft radius (Hiroshima, 2016). The $\delta_{\mathrm{FIT}}$ between the 3DCF disk and POM ring was determined to be 0.34 and that between the POM ring and shaft was $0.03 \mathrm{~mm}$. The outer radius of the POM ring at room temperature was set at $21.34 \mathrm{~mm}$ by adding $\delta_{\text {FIT }}(0.34 \mathrm{~mm})$ to the 3DCF disk's inner radius of $21 \mathrm{~mm}$. This outer radius shrinks to 20.87 $\mathrm{mm}$ upon exposure of the ring to liquid nitrogen; this radius is smaller than the inner radius of the 3DCF disk. The shrunk POM ring can be inserted into the center hole of the 3DCF disk. Although the inner radius of the POM ring is larger than the outer radius of the shaft before POM's cooling fit into the 3DCF disk, it becomes $0.03 \mathrm{~mm}$ smaller than the outer 
radius of the shaft at room temperature. In this situation, the shaft was press fit into the center hole of the POM ring at room temperature.

Figure 10 shows the time-dependent compressive stress on the POM ring-3DCF disk interface without rotation. The horizontal axis shows time (day). The latitude shows compressive normal stress $\sigma_{\mathrm{r}}$ in the radial direction. After three years, the stress became nearly constant. Viscoelastic analysis indicates that the normal stress $\sigma_{\mathrm{r}}$ increases from 3 to $11 \%$ after three years depending on temperature. After analyzing the viscoelasticity for three years, the MCKTS was calculated by a static analysis.

Figure 6 (b) shows the distribution of normal stress $\sigma_{\mathrm{r}}$ at a tip speed of $900 \mathrm{~m} / \mathrm{s}$. The $\sigma_{\mathrm{r}}$ on the interfaces of the two different materials of the three components varies from -38.8 to $-5.47 \mathrm{MPa}$. This result indicates that the three components remain in contact at this speed.

Figure 11 shows the temperature-dependent MCKTS of the POM ring. According to this figure, the MCKTS is predicted to not substantially degrade even after three years of using the POM ring. Though the disk has a predicted tip speed of $1520 \mathrm{~m} / \mathrm{s}$ for its material strength, it is reduced to $1284 \mathrm{~m} / \mathrm{s}$ because of the limit of POM's compressive stress of 70MPa. Viscoelastic effects after three years decreased the possible tip speed to $1210 \mathrm{~m} / \mathrm{s}$ at $25^{\circ} \mathrm{C}$. This is the case when the POM ring is hollow. Figure 12 shows the temperature-dependent MCKTS when a POM disk without hole is used. In case of the solid disk, MCKTS without viscoelastic effect is $1557 \mathrm{~m} / \mathrm{s}$, exceeding $1520 \mathrm{~m} / \mathrm{s}$ which is estimated by its material strength. Compared with a hollow disk, a solid disk produces small compressive stress under equivalent outer diameters when cooling-fitted. The solid POM disk improves the MCKTS to $1456 \mathrm{~m} / \mathrm{s}$ at $25^{\circ} \mathrm{C}$. Similarly, a smaller center hole in the POM ring is also expected to improve the MCKTS.

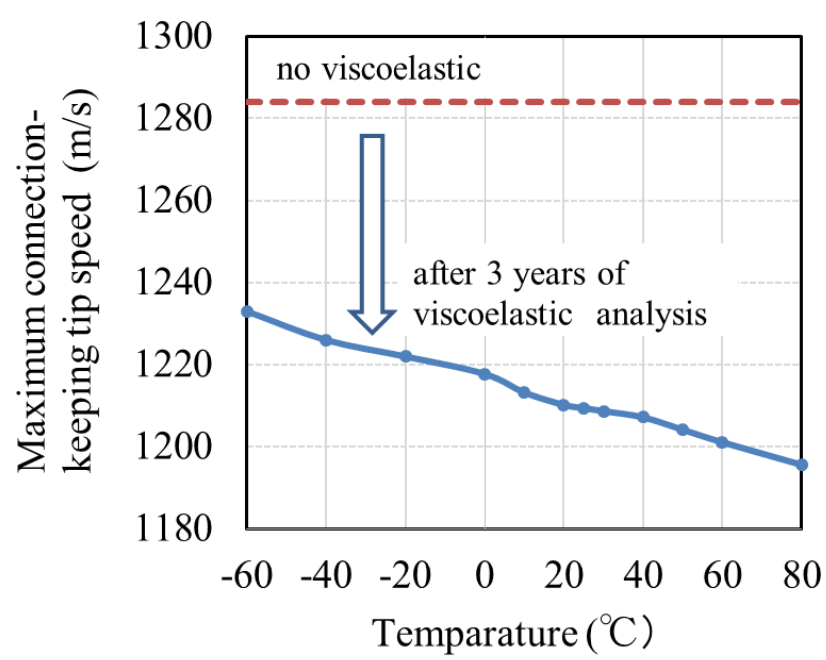

Fig.11 Temperature-dependent maximum tip speed up to which the interfacial connection between POM ring and $3 \mathrm{DCF}$ disk is stable.

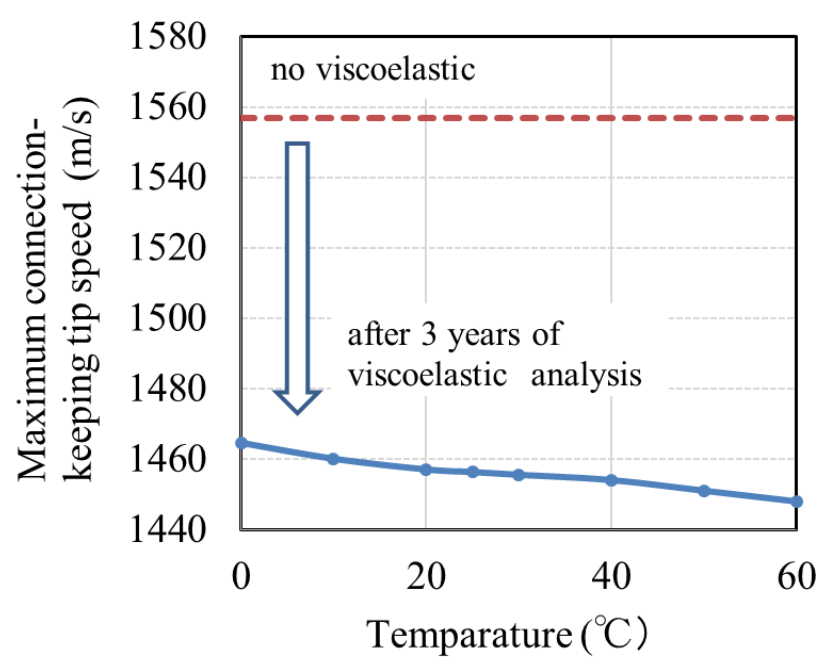

Fig.12 Temperature-dependent maximum tip speed up to which the interfacial connection between solid POM cylinder and 3DCF disk is stable.

\subsection{Spin tests}

Figure 13 shows the maximum amplitude during a spin test measured at point $U$ in Fig. 4. The horizontal axis shows the rotational speed (rpm). In the figure, a small peak at $600 \mathrm{rpm}$ resulted from first-order resonance. The second peak was caused by self-excitation at $6000 \mathrm{rpm}$. This excitation ceased at approximately 10,000 rpm, and the amplitude gradually increased thereafter. The spin test was stopped at 56,700 rpm (908 m/s) because the amplitude exceeded the danger threshold of $150 \mu \mathrm{m}$. Temperature of the tested rotor inside the camber rose from $26.0^{\circ} \mathrm{C}$ at the start of the spin test to $38.8^{\circ} \mathrm{C}$ in the end because of the slight air friction during the rotation.

\subsubsection{Fourier transformed amplitude}

Figure 14 presents the result of Fourier transformed amplitude up to 30,000 rpm, as shown in Fig. 13. In Fig. 14, the horizontal axis represents frequency $(\mathrm{Hz})$, the axis in the depth direction shows rotational speed, and the vertical axis shows intensity. Two characteristic intensity patterns are observed in the figure: synchronous with frequency and 
asynchronous.

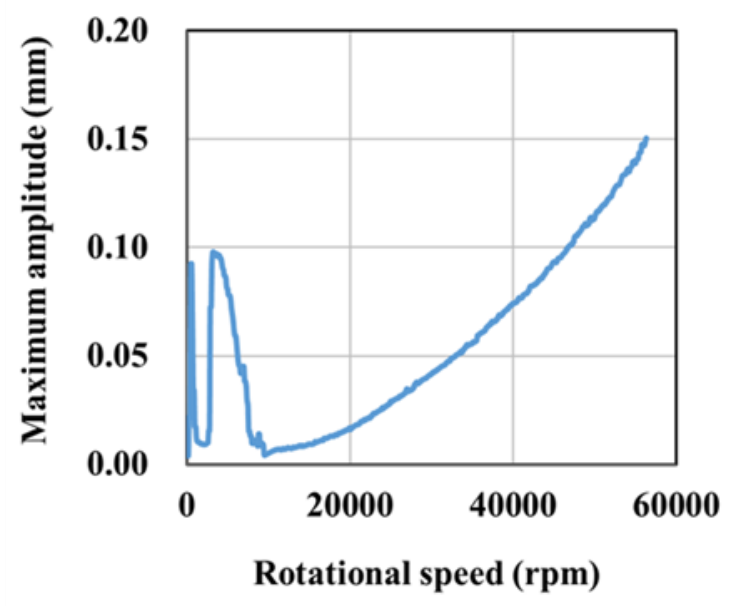

Fig. 13 Rotational speed and measured maximum amplitude of the shaft at point $\mathrm{U}$ during a spin test.

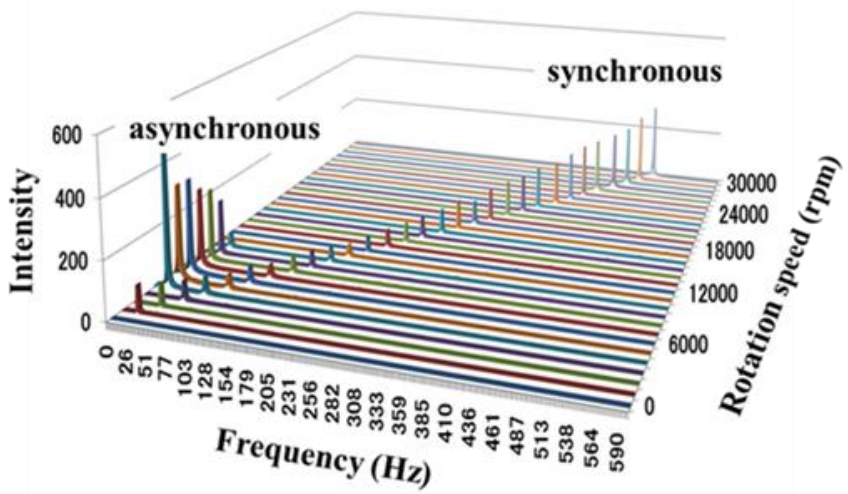

Fig. 14 Vibrational spectra measured at point $\mathrm{U}$ during a spin test.

The asynchronous intensity is composed of frequencies from 18 to $26 \mathrm{~Hz}$, which approach the frequency of the specimen's first-order resonance. This suggests that the asynchronous intensity pattern was caused by self-excited vibrations. A self-excited vibration is defined as a phenomenon in which friction in a sliding part intermittently supplies energy to a vibration system and enhances its amplitude (Den Hartog, 1956). This phenomenon was not problematic for high-speed rotation because it disappeared at higher rotation speeds.

The synchronous amplitude increased with frequency, especially at rotation speeds beyond 10,000 rpm. This suggests that this amplitude increase was caused by rotational mass unbalance in the specimen. Although the mass unbalance including test fixtures was minimized, the intrinsic mass unbalance of $19.3 \mathrm{~g} \cdot \mathrm{mm}$ in the 3DCF disk might provide vibration force during high-speed rotation and increase its amplitude.

\subsubsection{Experimental confirmation of possible MCKTS}

When the 3DCF disk exhibits its full potential stiffness based on the rule of mixture, the MCKTS becomes $1210 \mathrm{~m} / \mathrm{s}$ at $25^{\circ} \mathrm{C}$ (Fig. 11). If the stiffness degrades because of, for example, the curvature of fibers, the disk's inner radius easily expands by centrifugal force. Thus, compressive stress on the interface is immediately cancelled, resulting in separation of the POM ring and 3DCF disk. To evaluate the stiffness of the 3DCF disk, its displacement was measured at the outer radius and the result was compared with that obtained by FEA calculation. The ratio between the measured and calculated radii might provide information about the extent to which the stiffness of the disk was degraded.

Displacement at the outer radius of the disk includes the dynamic vibration amplitude and $\theta$-independent homogeneous expansion by centrifugal force. The expansion (displacement) during rotation can be measured by a pair of laser sensors (points P and Q) placed at two opposite peripheries, as shown in Fig. 4. An average of the two sensors cancels dynamic vibration, providing only the expansion measurement. Figure 15 presents a comparison of the measured and calculated outer radius displacements. As this figure shows, the measured expansion is approximately 1.35 times larger than the calculated expansion, meaning that the measured stiffness at the outer radius is $74 \%$ of the calculated stiffness. When the stiffness decreases to $74 \%$ of the design, the tip speed that gives the same displacement also decreases. Deformation is, in general, proportional to the square of the tip speed. The degraded MCKTS can be estimated to be 1040 $\mathrm{m} / \mathrm{s}(1210 \mathrm{~m} / \mathrm{s} \times \sqrt{ } 0.74)$. The spin test was stopped at a tip speed of $908 \mathrm{~m} / \mathrm{s}$ because of the risk of unexpected rotor fracture as a result of weakening compressive stress, which might loosen the interface and causing the POM ring to slip around the disk.

Nasu et al. (2002) reported a spin test of a 3DCF disk to a tip speed of $825 \mathrm{~m} / \mathrm{s}$. The tip speed of $908 \mathrm{~m} / \mathrm{s}$ in the present study is substantially greater than that in their study and confirms the validity of using a POM ring as a hub for high-speed rotation.

\subsubsection{Rotating conditions}

Under rotation, bending of the shaft and spindle can be considered to be the main mode of vibration. The spindle 
diameter is $7.9 \mathrm{~mm}$, which is smaller than that of the shaft $(22 \mathrm{~mm})$ as shown in Fig.2. Thus, the shaft is much stiffer than the spindle; consequently, we assumed the shaft to be a rigid body. Only the spindle bends to generate vibration. Under this assumption, the shaft rather tilts from the rotational axis and maintains a straight line during rotation (Hiroshima, 2016). This tilt can be observed as two different displacements at points U and L.

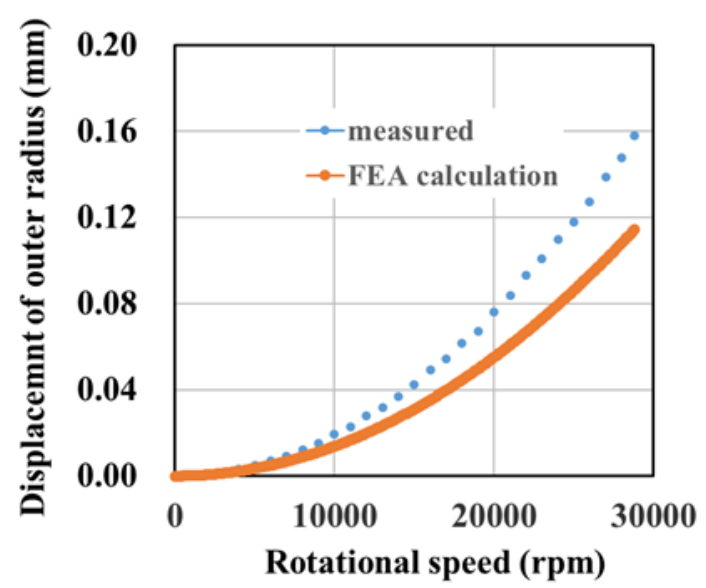

Fig. 15 Comparison of measured outer radius displacements with that calculated one by FEA.

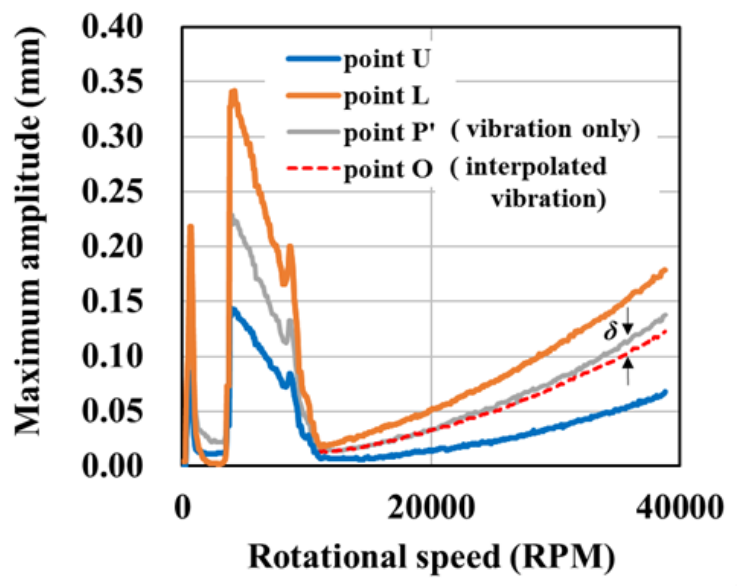

Fig. 16 Rotational speed and measured maximum amplitude measured at various locations during a spin test. The value represented by point $\mathrm{P}$ ' excludes disk expansion from that at point $\mathrm{P}$.

While the displacement at point $\mathrm{P}$ includes the vibration amplitude and disk expansion by centrifugal force, that at point $\mathrm{O}$ includes only the vibration amplitude. Although the vibration amplitude at point $\mathrm{O}$ cannot be measured directly using a sensor, it can be obtained by linear interpolation of the displacements at points $U$ and $L$. The amplitudes at points $\mathrm{O}$ and $\mathrm{P}$ are expected to be the same provided the 3DCF disk expansion by centrifugal force is excluded from point $\mathrm{P}$ and asymmetric deformation does not occur in the radial direction (Hiroshima, 2016).

Figure 16 shows the maximum amplitude in the radial direction measured at points $\mathrm{U}, \mathrm{L}, \mathrm{P}$ ' and $\mathrm{O}$ as a function of rotational speed. point $\mathrm{P}$ ' is the value obtained after excluding the 3DCF disk expansion from the measured value at point P. Consequently, point $\mathrm{P}^{\prime}$ represents only the vibration amplitude. The results in Fig. 16 show that the amplitudes of the three points increase in the order $\mathrm{U}<\mathrm{P}^{\prime}<\mathrm{L}$ at rotational speeds exceeding 10,000 rpm. The dashed line in the figure is a prediction at point $\mathrm{O}$ obtained by linear interpolation. Because point $\mathrm{P}$ ' is located between $\mathrm{U}$ and $\mathrm{L}$ in the axial direction (Fig. 4), vibration measured at point $\mathrm{P}^{\prime}$ agrees with that measured at point $\mathrm{O}$ if asymmetric deformation does not occur in the radial direction. point $\mathrm{P}^{\prime}$ in Fig. 16 began to become larger than point $\mathrm{O}$ at $20,000 \mathrm{rpm}$. This result might stem from the 3DCF disk's inherent mass unbalance that induces asymmetric in-plane deformation by centrifugal force and further mass unbalance.

Asymmetric in-plane deformation induced by inherent mass unbalance in the 3DCF disk is represented as $\delta$ in Fig. 16. This $\delta$ quantity was estimated by FEA. Figure 17(a) shows the model in which 56,748 elements and 247,336 nodes constitute a half-disk. Mass elements of $19.3 \mathrm{~g} \cdot \mathrm{mm}$ in total, equivalent to the inherent mass unbalance, were added on the right side of the cross section of the 3DCF disk. Other FEA conditions were the same as those in the model in Fig. 6. Figure 17(b) presents the FEA result of asymmetric radial displacement at 38,000 rpm. This figure shows a larger shift of the disk's outer radius toward the right. Displacements are $0.2568 \mathrm{~mm}$ on the right side and $0.2303 \mathrm{~mm}$ on the left side. The half-difference between the two sides $(0.0132 \mathrm{~mm})$ constitutes $\delta$, which is close to $\delta(0.014 \mathrm{~mm})$ in Fig. 16 . This suggests that the inherent mass unbalance in the 3DCF disk most likely induced the asymmetric in-plane deformation and produced further mass unbalance. Consequently, with increasing rotational speed, the rotor's vibration amplitude was gradually increased by the unbalanced mass. 

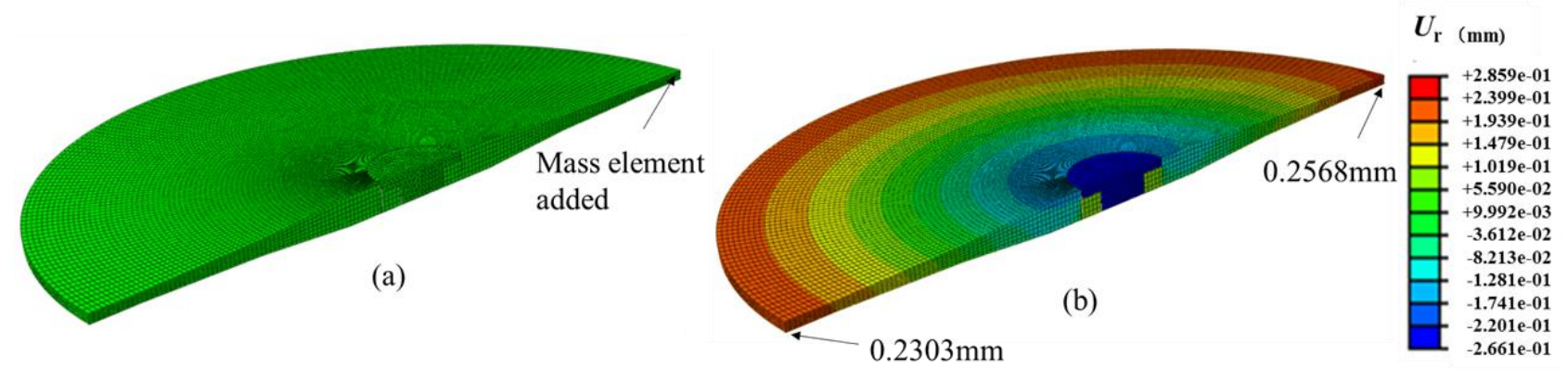

Fig. 17 A half FEA model for the trial rotor: () perspective view and (b) asymmetric radial displacement $\left(U_{\mathrm{r}}\right)$ at $38,000 \mathrm{rpm}$ induced by mass imbalance embedded in the right-hand side of the disk.

\section{Discussion}

One method for improving the rotational speed limit is minimizing the inherent mass unbalance in the 3DCF disk. A lower inherent mass unbalance in the disk might be less affected by centrifugal force and produce less eccentricity. Voids remaining in the disk are likely the cause of the mass unbalance. The disk has a porosity of $1.8 \%$. One approach to reducing porosity is to infiltrate the resin into the fabric under high pressure.

Another approach is applying a higher compressive stress $\sigma_{r}$ during the cooling fit process. A larger compressive stress enhances the MCKTS. The $\sigma_{r}$ induced by the same amount of $\delta_{\text {FIT }}$ depends on the hole diameter of the POM ring. In fact, the MCKTS varies from Fig. 11 to 12 depending on the outer radius of the shaft shown in Fig. 2. A solid disk (without a hole) maximizes the MCKTS.

A third approach is to enhance the disk stiffness. Because a disk with high stiffness undergoes less expansion of its inner radius, compressive stress induced by cooling fit remains sufficiently high at high rotation speeds to keep the interface connection stable. FEA shows that high disk stiffness diminishes in-plane deformation against mass unbalance and thereby reduces vibration amplitude.

\section{Conclusions}

The method of connecting a driving shaft to a disk via a POM ring is effective for a high-speed rotating 3DCF disk with a small inner-to-outer radius ratio. To demonstrate the validity of this approach, we fabricated a trial spin test rotor in which a 3DCF disk comprising $r$-, $\theta$-, and $z$-direction-reinforcing fibers and a driving shaft were assembled with a POM ring. The spin tests of the trial rotor were conducted at $908 \mathrm{~m} / \mathrm{s}$ without fracture, the highest ever tip speed achieved using a 3DCF disk with a maximum amplitude of $150 \mu \mathrm{m}$. Finally, we suggested methods for further improving the tip speed.

\section{Acknowledgment}

This work was partly supported by the Space-Lab project, sponsored by the Japan Aerospace Exploration Agency.

\section{References}

Arvin, A. C. and Bakis, C. E., Optimal Design of Press-fitted Filament Wound Composite Flywheel Rotors, Compo Struct, Vol.72, (2006), PP.47-57.

Den Hartog, J.P., Mechanical Vibration $4^{\text {th }}$ Ed, McGRAW-HILL Book Company, (1956).

Gabrys, C.W. and Bakis, C.E., Design and testing of composite flywheel rotors, In : ASTM STP-1242, (1997), PP.3-22.

Goto, K., Hatta, H., Kogo, Y., Fukuda, H., Sato T and Tanatsugu, N., Carbon-carbon composites turbine disk for the air turbo ramjet engine (ATREX), Adv Composite Mater, Vol.12, No.2, (2003), PP.205-222.

Gowayed,Y., ABEL-HADY, F., Flowers, G.T. and Trudell, J.J., Optimal design of multi-direction composite flywheel rotors. Polymer Composites, Vol.23, No.3, (2003), PP.433-441.

Ha, S. K., Kim, M. H. and Han, S.C., Design and spin test of a hybrid composite flywheel rotor with a split type hub, J Compos Mater, Vol.40, No.23 (2006), PP.2113-2130.

Hayes, R. J., Kajs, J. P. and Thompson, R. C., Design and Testing of a Flywheel Battery for a Transit Bus, Society of Automotive Engineers, Inc., (1998).

Hiroshima, N., Hatta, H., Masashi, K., Goto, K., G. and Kogo, Y., Optimization of flywheel rotor made of three- 
dimensional composites, Compo Struct, Vol.131, (2015), PP.304-311.

Hiroshima, N., Hatta, H., Koyama, M., Yoshimura, J., Nagura, Y., Goto, K. and Kogo, Y., Spin test of three-dimensional composite rotor for flywheel energy storage system, Compo Struct, Vol.136, (2016), PP.626-634.

JIS 0905 or ISO1940-1, (1986).

JIS K7181 (2011) or ISO604.

JIS K7244 (1998) or ISO6721.

Nasu, H. and Hirose, M., Research and development of three-dimensional fabric flywheel, Shikoku sougou kennkyusho reports, Vol.79, (2002), PP.78-85. (in Japanese).

Ogasawara, T., Yoshimura, A. and Kamita, T., Tensile strength of a threaded screw hole in plain woven carbon fiber/epoxy, JAXA Research and Development Memorandum, JAXA-RM-11-002, (2012). (in Japanese).

Sakai, T., Linear viscoelastic creep analysis for thermosetting resin and the composites. PhD Thesis, University of Keio, Tokyo, Japan, (2008).

Takahashi, K., Kitade, S. and Morita, H., Development of high speed composite flywheel rotors for energy storage system, Adv Composite Mater, Vol.11, No.1, (2002), PP.41-50.

Toray Carbon Fiber America, Decatur, Alabama, USA.

Yoshimura, J., Hiroshima, N. and Hatta, H., Development of high speed rotation disk made of carbon fiber reinforced three-dimensional composites, 14th ECCM, (2010). 